\title{
In Cystic Fibrosis Homozygotes and Heterozygotes, Neutrophil Apoptosis Is Delayed and Modulated by Diamide or Roscovitine: Evidence for an Innate Neutrophil Disturbance
}

\author{
Sandra Moriceau ${ }^{\mathrm{a}}$ Gérard Lenoir $^{\mathrm{b}}$ Véronique Witko-Sarsat ${ }^{\mathrm{a}, \mathrm{c}}$ \\ a INSERM U845 and bepartment of General Pediatrics, Necker Hospital, ' INSERM U1016-CNRS UMR \\ 8104-Université Paris Descartes, Cochin Hospital, Paris, France
}

\section{Key Words}

Apoptosis $\cdot$ Cystic fibrosis transmembrane regulator •

Diamide $\cdot$ Inflammation $\cdot$ Neutrophil $\cdot$ Roscovitine

\begin{abstract}
Cystic fibrosis (CF) is a chronic inflammatory lung disease characterized by polymorphonuclear neutrophil (PMN)dominated airway inflammation. Defective apoptosis might explain PMN persistence at these inflammation sites. We previously reported that in CF patients PMN underwent delayed apoptosis, which was not always related to their infectious state and independent of the type of CF transmembrane regulator (CFTR) mutation. To understand the role of infection and PMN apoptosis in CF, PMN apoptosis was investigated in CF parents who are obligate heterozygotes for the CFTR mutation but without chronic bacterial infection. They also demonstrated delayed PMN apoptosis compared with healthy controls, as assessed by annexin- $V$ labeling and caspase-3 cleavage. Diamide, a direct thiol-oxidizing agent, potentiated PMN apoptosis in controls and CF patients, resulting in similar levels of constitutive and Fas-potentiated apoptosis. The cyclin-dependent kinase inhibitor roscovitine provided another approach to restore normal PMN apoptosis. However, the selective CFTR inhibitor CFTR Inh172 $_{12}$ did not affect PMN apoptosis in control subjects. Apparent-
\end{abstract}

ly, the dysregulation of CF PMN is not only a consequence of the chronic infectious state in CF children but might also be related to CF 'intrinsic' factors. Restoration of normal PMN apoptosis by cellular redox modulation or roscovitine opens new research avenues to decrease PMN-mediated inflammation in CF.

Copyright $\odot 2010$ S. Karger AG, Basel

\section{Introduction}

Cystic fibrosis (CF) is an autosomal recessive disorder caused by mutation in the CF transmembrane regulator (CFTR) gene coding for a chloride channel. In the airways, this defect leads to an increased mucus viscosity and hypersecretion that impair mucociliary clearance, being ultimately responsible for chronic bacterial colonization. Hence, most CF-associated morbidity and mortality result from progressive pulmonary disease with lung parenchymal destruction and chronic pulmonary bacterial colonization $[1,2]$. One of the hallmarks of CF is massive polymorphonuclear neutrophil (PMN) influx into the airways that is tightly linked with clinical status, as shown by the strong correlation between pulmonary function test scores and PMN-derived proteinases [3]. Thus, airway inflammation typical of CF is based on the

\section{KARGER}

Fax +4161306 1234

E-Mail karger@karger.ch

www.karger.com
(C) 2010 S. Karger AG, Basel

$1662-811 X / 10 / 0023-0260 \$ 26.00 / 0$

Accessible online at:

www.karger.com/jin
Dr. Véronique Witko-Sarsat

INSERM U1016, Cochin Institute, Immunology-Hematology Department 27, rue du Faubourg Saint-Jacques

FR-75014 Paris (France)

Tel. +331 405166 56, Fax +33 1405165 35, E-Mail veronique.witko@inserm.fr 
paradox of excessive PMN recruitment and activities, and failure of their microbicidal activities, resulting in chronic Pseudomonas aeruginosa infection. Hence, the extraordinary numbers of PMN accumulating within airways of CF patients has led to the hypothesis of an innate immunity failure $[4,5]$.

Previous studies on CF patients' PMN indicated functional disturbances in bacterial phagocytosis and killing, and other effector functions [6]. Because of the extreme heterogeneity of CF patients in terms of the infectious status, comparisons of experiments and results are difficult. At present, the generally accepted conclusion is that circulating PMN are primed and pre-activated because of chronic or undetectable infection $[7,8]$. Using a gene microarray approach, differences in gene expression patterns of PMN were found between clinically stable and non-infected CF patients and healthy controls. The difference in 'resting' PMN between controls and CF patients exceeded that between the latter and inflammatory' PMN from bronchoalveolar lavage fluids. These findings are consistent with a perturbed 'inflammatory program' in CF PMN, which remains to be investigated in further studies to elucidate possible disturbances [9].

However, the knotty point remains to discriminate between infection-driven and 'constitutive' inflammation in CF patients. One approach to try to resolve this issue was to study PMN from CF parents heterozygous for a CFTR mutation and free of chronic bacterial infection and clinical symptoms. We previously reported a significant increase in myeloperoxidase-dependent oxidant formation in both CF children and their parents, in comparison to healthy controls, whereas NADPH oxidase activity was similar to that of controls. The fact that this increase was observed in the parents of CF children led us to conclude that the impaired myeloperoxidase activity observed in CF was constitutive, i.e. independent of infection, and reflected altered $\mathrm{pH}$ regulation in CF PMN [10]. Studying cells from heterozygous subjects might provide unique cellular models to understand the impact of CFTR mutation on a given function. Indeed, a study on chloride transport in nasal ciliated cells of CF heterozygotes provided evidence that one third of the subjects had very low levels of CFTR function, which were comparable to those found in CF patients [11]. This diminished function was not associated with more CF-like symptoms in heterozygous subjects but was strongly associated with the respiratory status of CF patients.

PMN apoptosis is a key step in inflammation resolution, and integrity of the apoptotic pathways is of paramount importance in $\mathrm{PMN}$-dominated inflammation
$[12,13]$. Given the number of PMN in CF airways, late PMN apoptosis could have devastating consequences. Indeed, we [14] and others [15] reported that PMN from CF patients undergo delayed apoptosis, thereby slowing their removal by macrophages and potentiating airway inflammation. In the latter study, delayed apoptosis was obtained by incubating CF patient serum with PMN from healthy subjects suggesting an acquired rather than a constitutive mechanism. The authors identified C-reactive protein as the causative agent that delayed PMN apoptosis [15]. Decreased levels of the pro-apoptotic protein Bax [16] or increased levels of prosurvival proteins, such as coronin-1A [14], in PMN from CF patients have been discovered. Since PMN functions are markedly dependent on the inflammatory environment and the infectious state, it is extremely difficult to discern whether disturbed PMN function is a consequence of activation or an innate defect. The aim of this report was to provide some insights into the molecular mechanisms of late apoptosis in CF. Therefore, we investigated PMN from CF heterozygotes and found delayed PMN apoptosis, highly suggestive of an innate defect in the control of cell survival.

\section{Patients and Methods}

\section{PMN Isolation}

PMN were isolated from the EDTA-anticoagulated blood of healthy volunteers (Etablissement Français du Sang, Paris, France), or CF patients or parents $(n=19)$ using density gradient centrifugation (Polymorphprep; Nycomed), as described previously [17]. Thirty-two CF patients, 15 girls and 17 boys (mean age \pm SEM: $12.4 \pm 0.85$ years), treated in the Department of General Pediatrics (Necker Enfants Malades Hospital, Paris, France) were recruited for the study. CF was diagnosed according to standard criteria, including a sweat test. Genotyping of CF patients showed the following allelic frequencies: $52 \% \Delta F 508$ homozygotes, $15 \% \Delta F 508$ heterozygotes with an unknown mutation, and $33 \%$ with other mutations in both alleles. All CF patients had chronic $P$. aeruginosa or Staphylococcus aureus colonization but were in stable clinical condition. Patients or their parents gave written informed consent to participate in the study. This study was approved by the Ethics Committee of the Necker Enfants Malades Hospital.

\section{Apoptosis Induction and Quantification}

To induce apoptosis, PMN were resuspended at $2 \times 10^{6}$ cells/ $\mathrm{ml}$ in RPMI (Gibco) supplemented with $10 \%$ fetal calf serum. After $16 \mathrm{~h}$ of incubation at $4^{\circ} \mathrm{C}, \mathrm{PMN}$ were incubated for the indicated times at $37^{\circ} \mathrm{C}$ in a humidified atmosphere with $5 \% \mathrm{CO}_{2}$ without any exogenous stimulus to induce constitutive apoptosis or with anti-Fas monoclonal antibody (10 ng/ml, Beckman-Coulter) or with gliotoxin $(2 \mu \mathrm{g} / \mathrm{ml}$, Sigma) to stimulate the death re- 
ceptor pathway and accelerate apoptosis. When indicated, the selective CFTR inhibitor CFTR Inh172 (a kind gift from Frédéric Beck, University of Poitiers), diamide (Sigma) or roscovitine (Calbiochem) was added at the indicated concentrations.

Apoptosis-induced phosphatidylserine externalization was quantified by annexin-V labeling, excluding necrotic 7-amino actinomycin D (7AAD)-labeled cells, as previously described [17]. Briefly, $5 \times 10^{5} \mathrm{PMN}$ were incubated in $100 \mu \mathrm{l}$ of annexin- $\mathrm{V}$ buffer (Becton-Dickinson) containing $10 \mu \mathrm{l}$ of 7AAD and $5 \mu \mathrm{l}$ of phycoerythrin (PE)-conjugated annexin- $\mathrm{V}$ for $15 \mathrm{~min}$ at room temperature. Cells were then resuspended in $300 \mu \mathrm{l}$ of annexin-V buffer and analyzed by flow cytometry for annexin-V-PE (FL2) and 7AAD (FL3) labeling, using a FACScan flow cytometer (Cell Quest software and Becton-Dickinson immunocytometry systems). Caspase activation was assessed by Western blot analysis, using a polyclonal anti-caspase- 3 antibody as the primary antibody (Santa Cruz), which specifically recognizes the cleaved 17kDa caspase-3 fragment.

\section{Statistical Analysis}

Statistical analysis was performed using Statview software. The $\mathrm{p}$ value was determined with Student's t test.

\section{Results and Discussion}

\section{Delayed PMN Apoptosis in CF Patients and in Their Parents}

As shown in figure 1a, physiologic PMN apoptosis, assessed as the percentage of PMN with externalized phosphatidylserine, occurred later in CF children's PMN than control PMN. The kinetic study showed significantly delayed apoptosis of CF PMN compared with controls from 1 to $15 \mathrm{~h}$ of incubation. We previously demonstrated that this later phosphatidylserine externalization was associated with a later mitochondrial depolarization [14]. However, this delayed apoptosis was not associated with a previous $P$. aeruginosa infection (data not shown). To understand the contributions of infection and intrinsic PMN disturbance, $\mathrm{PMN}$ apoptosis was investigated in CF parents, who are obligate heterozygotes for the CFTR mutation but without chronic bacterial infection. Herein, we provide evidence of delayed apoptosis of PMN from CF parents, compared with controls, using annexin-V labeling and caspase- 3 cleavage. Accordingly, the abilities of PMN to undergo apoptosis did not differ between CF children and their parents, thus strongly suggesting an innate PMN perturbation in CF. This late phosphatidylserine externalization was concomitant with delayed caspase- 3 activation in PMN of both CF children and their CF parents, compared with controls, which was confirmed by Western blot analysis of the cleaved caspase-3 fragment, being absent in CF children and revealing only very faint bands in CF parents, in contrast to the strong bands in controls (fig. 1b).

We next hypothesized that stimulation of the death receptor pathway might restore a normal level of PMN apoptosis. This was not the case, because, as shown in figure 1c, anti-Fas-potentiated PMN apoptosis was also delayed in CF patients and CF parents compared with controls. Moreover, PMN apoptosis triggered by gliotoxin, which induces Bak dimerization [18] and interferes with the NF- $\kappa$ B pathway [19], was also delayed in CF patients compared with controls (fig. 1d). Hence, this late PMN apoptosis was observed regardless of the apoptosis pathway, either constitutive or via death receptors, or the mitochondrial pathway, suggesting that the limiting step was a common step in these pathways. Notably, PMN counts were higher in CF patients $\left(3.77 \pm 0.38 \times 10^{6}\right.$; $\mathrm{p}<0.001)$ and in CF parents $\left(3.97 \pm 0.45 \times 10^{6}, \mathrm{p}<\right.$ $0.001)$ compared with controls $\left(1.54 \times 10^{6} \pm 0.11\right)$, thus strongly suggesting that mechanisms intrinsic to $\mathrm{CF}$ seem to promote PMN survival or affect their physiologic turnover.

Diamide, a Direct Thiol-Oxidizing Agent, Potentiated Apoptosis of CF Patient PMN and Restored Normal PMN Apoptosis

The next step was to identify signaling pathways whose stimulation could promote apoptosis and reverse the delayed apoptosis of CF PMN. It was previously shown that a redox status change is an early, ubiquitous event in PMN apoptosis, and could be considered the trigger for cell death [20]. Diamide is a thiol-oxidizing agent, which induces apoptosis via the mitochondrial pathway and caspase-3 activation. Sensitivity to diamide is correlated with a lower GSH/GSSG ratio; pertinently, caspase-3 activation is an important step in GSH depletion-induced apoptosis in resting and inflammatory PMN. Moreover, the inhibitory effect of lipopolysaccharides and granulocyte macrophage colony-stimulating factor on spontaneous PMN apoptosis was reversed by diamide, which appears to be able to overcome 'survival signals' [21]. As described above, PMN apoptosis occurred late in CF patients compared with controls. We confirmed that diamide $(200 \mu \mathrm{M})$ significantly enhanced PMN apoptosis without inducing necrosis (data not shown). Notably, in the presence of diamide, no significant difference in PMN apoptosis was observed between CF patients and controls (fig. 2). Likewise, diamide reversed the delayed Fas-potentiated apoptosis of CF PMN, thus strongly suggesting that diamide is able to overcome the 'survival signal' in CF. 


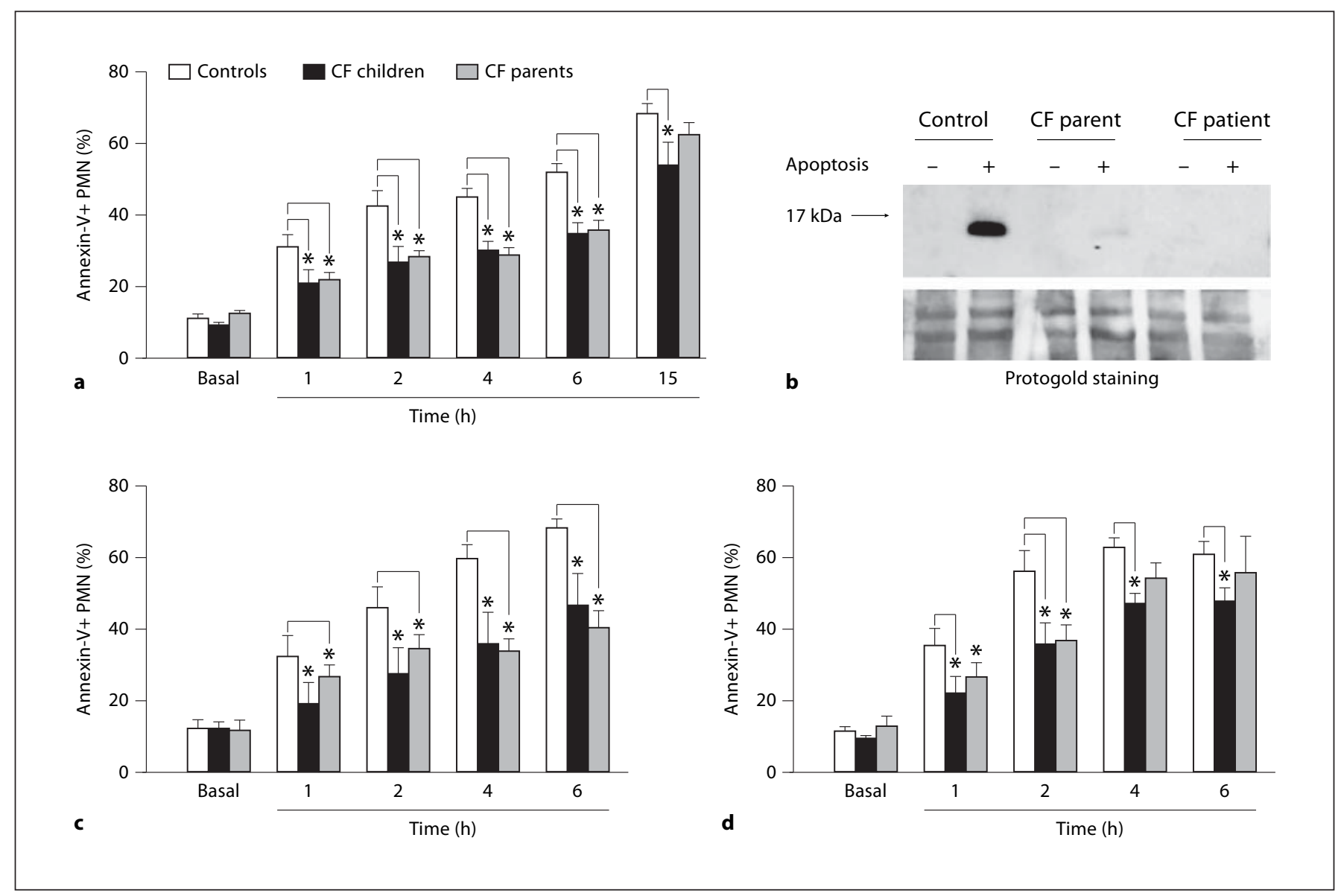

Fig. 1. Analysis of spontaneous PMN apoptosis in CF. a Phosphatidylserine externalization on PMN from CF children and their parents. $\mathrm{PMN}$ were incubated at $37^{\circ} \mathrm{C}$ for the indicated times and phosphatidylserine externalization was detected using PE-conjugated annexin-V labeling. Basal represents the level of apoptosis just before incubation at $37^{\circ} \mathrm{C}$. Data are percentages of annexin$\mathrm{V}$-positive PMN and are expressed as means \pm SEM for PMN from controls $(n=29)$, CF patients $(n=31)$ and CF parents $(n=$ $21 ;{ }^{*} \mathrm{p}<0.05$, using ANOVA). $\mathbf{b}$ Western blot analysis of active caspase-3 in PMN from a CF patient and his CF parent and a control. Isolated $\mathrm{PMN}$ were incubated for $4 \mathrm{~h}$ at $37^{\circ} \mathrm{C}$ and then lysed by sonication $(50 \mu \mathrm{g} /$ well) for Western blot analysis with a polyclonal anti-active caspase- 3 antibody. Protogold staining of the membrane is shown as the loading control. In this representative

\section{The Cyclin-Dependent Kinase Inhibitor Roscovitine \\ Reversed Delayed Apoptosis of CF PMN}

Another novel pharmacological approach recently described to trigger PMN apoptosis is the use of the cyclindependent kinase (CDK) inhibitor roscovitine [22]. Those authors reported that CDK activities, so far restricted to cell-cycle regulation, can control apoptosis in nonproliferating cells, such as PMN. Indeed, roscovitine was experiment, the respective percentages of annexin-V-positive PMN were 47,10 and $25 \%$ for the control, CF patient and CF parent, respectively. c Analysis of anti-Fas-induced PMN apoptosis in CF. PMN were incubated at $37^{\circ} \mathrm{C}$, as in a, except that anti-Fas monoclonal antibody $(1 \mu \mathrm{g} / \mathrm{ml})$ was added to stimulate the deathreceptor-dependent apoptosis pathway. Phosphatidylserine externalization was detected using PE-conjugated annexin-V labeling. Basal represents the level of apoptosis just before incubation at $37^{\circ} \mathrm{C}$. Data are percentages of annexin-V-positive PMN and are expressed as means \pm SEM for PMN from controls $(n=7)$, CF patients $(n=6)$ and CF parents $\left(n=6{ }^{*} p<0.05\right.$, Student's t test). d Analysis of gliotoxin-induced PMN apoptosis in CF (see c, except that PMN apoptosis was induced by $2 \mu \mathrm{g} / \mathrm{ml}$ gliotoxin). shown to enhance inflammation resolution in animal models of airway inflammation and arthritis. Although molecular mechanism underlying this pro-apoptotic effect in PMN have not been completely elucidated, an involvement of caspase-3 activation has been put forward. Roscovitine also improved inflammation resolution after experimental pneumococcal infection and accelerated recovery [23], suggesting that roscovitine combined with 


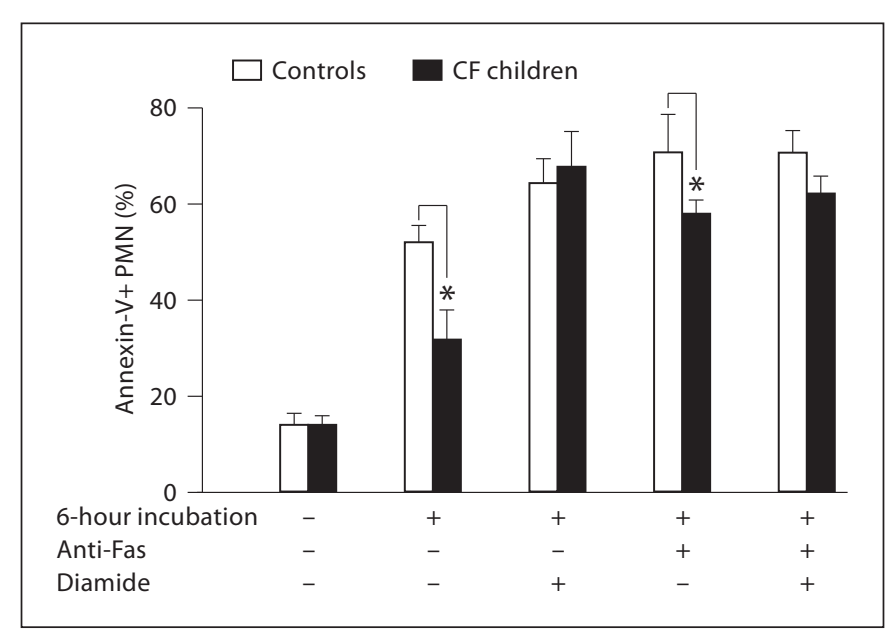

Fig. 2. The thiol-oxidizing agent diamide restored normal levels of CF PMN apoptosis. Constitutive apoptosis was induced by incubation at $37^{\circ} \mathrm{C}$ for $6 \mathrm{~h}$ without any modulating agent, with diamide or anti-Fas, or both. Phosphatidylserine externalization was detected using PE-conjugated annexin-V labeling. Data are the percentages of annexin-V-positive PMN and are expressed as means \pm SEM for PMN from controls $(n=7)$ and CF patients ( $\mathrm{n}=7{ }^{*} \mathrm{p}<0.05$, Student's t test).

antibiotics might also be beneficial in the treatment of $\mathrm{CF}-\mathrm{associated} \mathrm{lung} \mathrm{disease.} \mathrm{Herein,} \mathrm{we} \mathrm{confirmed} \mathrm{that}$ roscovitine induced apoptosis of control PMN in a dosedependent manner. Interestingly, roscovitine used at 10 $\mu \mathrm{M}$ restored normal apoptosis levels for CF PMN, thus overcoming the CF 'survival signal' (fig. 3).

\section{Lack of an Effect of the Selective Inhibitor CFTR Inh172 $_{2}$ on Neutrophil Apoptosis}

Despite some controversies concerning the functional expression of the CFTR gene in mature PMN, its encoded protein has been detected by Western blotting, raising the possibility that some PMN inflammatory features could result from the genetic defect [24]. To test whether CFTR channel activity could be involved in PMN constitutive apoptosis, the effect of the selective CFTR inhibitor $\mathrm{CFTR}_{\text {Inh172 }}$ on PMN apoptosis was examined (fig. 4). This agent had no effect on PMN apoptosis at concentrations that effectively inhibited CFTR activity in epithelial cells [25], thus arguing against the direct involvement of CFTR channel activity in this process.

The issue of whether the late CF PMN apoptosis is linked to CFTR is difficult to solve. The absence of an effect of the selective CFTR inhibitor argued against this possibility, but CFTR might have some functions distinct from its channel activity, such as its binding to key pro-

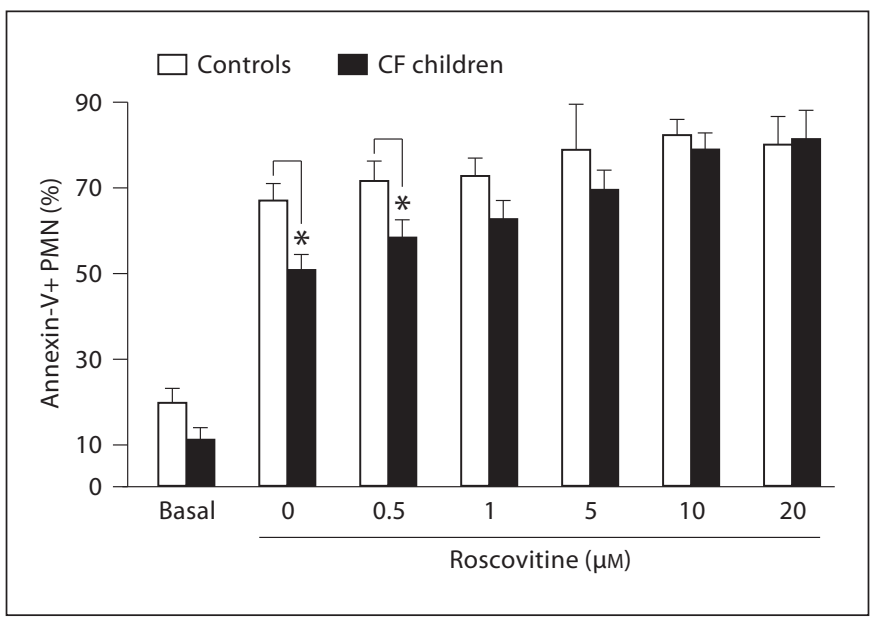

Fig. 3. The CDK inhibitor roscovitine restored normal levels of $\mathrm{CF}$ PMN apoptosis. Isolated PMN were incubated at $37^{\circ} \mathrm{C}$ for $15 \mathrm{~h}$ to induce constitutive apoptosis without or with increasing roscovitine concentrations. Phosphatidylserine externalization was detected using PE-conjugated annexin-V labeling. Data are the percentages of annexin-V-positive PMN and are expressed as means \pm SEM for PMN from controls $(n=10)$, and CF patients ( $\mathrm{n}=7 ;{ }^{*} \mathrm{p}<0.05$, Student's t test).

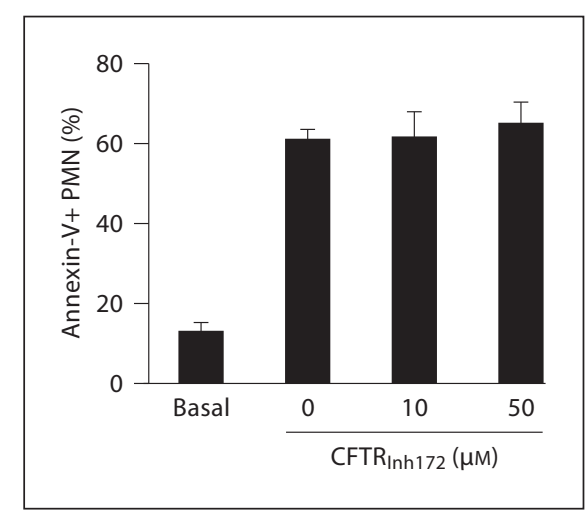

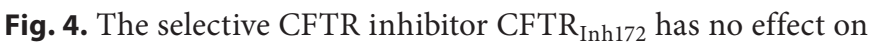
apoptosis of PMN from healthy subjects. PMN were incubated for $15 \mathrm{~h}$ at $37^{\circ} \mathrm{C}$ without or with increasing CFTR Inh172 $_{1}$ concentrations. Phosphatidylserine externalization was detected using PEconjugated annexin-V labeling. Data are means \pm SEM of 4 independent experiments. (No difference in PMN apoptosis was observed in the presence of the inhibitor.)

teins to regulate cell fate. Therefore, CFTR expression and function in mature PMN remain a matter of debate and require further investigation. Nonetheless, although CFTR function does not appear to be involved in the regulation of PMN apoptosis, some similarities with CFTRexpressing cells could be noted. In keeping with our PMN 
findings, the results of several studies showed that CFTR mutations inhibited apoptosis of different cell types [26, 27]. Moreover, an impaired redox status in CF cells, especially of GSH, the major cellular antioxidant, has been reported [28]. CFTR has been described as a GSH transporter able to trigger GSH efflux [29]. Notably, Fas-potentiated apoptosis is associated with GSH efflux and is mediated via caspase- 3 activation. Hence, this GSH efflux might be decreased in PMN from CF patients, thus indicating a CFTR defect. Diamide-induced neutrophil apoptosis is dependent on caspase and independent of factors that delay the apoptotic pathway, e.g. lipopolysaccharides or granulocyte macrophage colony-stimulating factor [30]. Although the molecular mechanisms leading to late neutrophil apoptosis in CF require further investigation, our data support the hypothesis that modulation of the redox potential by thiol-oxidizing agents promoting caspase- 3 activity might be used to promote apoptosis of CF PMN. In order to conciliate these novel data with our earlier findings on CF heterozygotes [10], it can be speculated that the late apoptosis in CF might be related to the excessive myeloperoxidase-dependent oxidant production that we have previously described in PMN from CF patients and their parents, since oxidants can block caspase activity [31].

CFTR cloning was a major milestone in understanding the molecular basis of CF, but dissecting CF pathogenesis has proven far more complicated. So far, mouse models have partly helped to unravel the pathophysiological mechanisms governing CF lung disease, but no animal model mimicking human pathology is available to date. At present, it is well recognized that effective management of airway inflammation could complement other therapies for CF. The non-steroidal anti-inflammatory agent ibuprofen led to a slower decline in pulmonary function and improved body weight, but this treatment was poorly tolerated. Moreover, ibuprofen treatment increased PMN activation at concentrations lower than the therapeutic range [32]. Although oral corticosteroids have shown a beneficial effect on CF lung disease, their side effects have limited the use of this therapy. In addition, in contrast to eosinophils, PMN have a higher survival rate in the presence of corticosteroids [33]. Clinical prognosis and lung disease severity are independent of the CFTR mutation, suggesting that other genetic defects might be involved in the complex pathogenesis of $\mathrm{CF}$, too. A recent genome-wide single nucleotide polymorphism scan approach in one CF cohort led to the identification of the histone-deacetylase-dependent transcriptional coregulator, interferon-related developmental regulator 1
(IFRD1), as a modifier gene in CF lung disease severity. Most interestingly, IFRD1 is expressed during terminal PMN differentiation. PMN, but not macrophages, from IFRD1-deficient mice had decreased effector function associated with lower NF-кB-p65 activation [34]. These new data indicate that PMN gene expression influences CF pathogenesis, thus corroborating the pivotal role of $\mathrm{PMN}$ as 'designers' shaping the inflammatory response and directly influencing the clinical outcome of patients.

Our data showed delayed PMN apoptosis in CF homozygotes and heterozygotes compared with control subjects, thus strongly suggesting an intrinsic CF-linked PMN dysfunction. This was in keeping with the increased PMN counts in both CF homozygotes and heterozygotes compared with controls. Moreover, it can be concluded that CFTR does not directly modulate PMN apoptosis via its chloride conductance activity. Our current hypothesis is that other CFTR functions, unknown modulatory factors or modifying genes intrinsic to CF might be involved in this delayed PMN apoptosis. Therefore, modulating $\mathrm{PMN}$ apoptosis to enhance inflammation resolution is an attractive therapeutic approach, which merits careful investigation. Indeed, it could be beneficial to use diamide or roscovitine to favor macrophage elimination of PMN. The molecular basis of late PMN apoptosis in CF remains to be defined, and examination of the mechanisms governing PMN clearance at the site of inflammation seems to be a relevant and promising research avenue in $\mathrm{CF}$.

\section{Acknowledgments}

This work was supported by research funding from the Association Mucoviscidose ABCF and European Life Sciences and Health Grant (LSHG-CT-2005-512044) NEUPROCF.

\footnotetext{
References $\quad 1$ Cantin A: Cystic fibrosis lung inflammation: early, sustained, and severe. Am J Respir Crit Care Med 1995;151:939-941.

-2 Nichols D, Chmiel J, Berger M: Chronic inflammation in the cystic fibrosis lung: alterations in inter- and intracellular signaling. Clin Rev Allergy Immunol 2008;34:146-162.

-3 Witko-Sarsat V, Halbwachs-Mecarelli L, Schuster A, Nusbaum P, Ueki I, Canteloup S, Lenoir G, Descamps-Latscha B, Nadel JA: Proteinase 3, a potent secretagogue in airways, is present in cystic fibrosis sputum. Am J Respir Cell Mol Biol 1999;20:729-736.

4 Bals R, Weiner DJ, Wilson JM: The innate immune system in cystic fibrosis lung disease. J Clin Invest 1999;103:303-307.
} 
5 Doring G, Gulbins E: Cystic fibrosis and innate immunity: how chloride channel mutations provoke lung disease. Cell Microbiol 2009;11:208-216.

6 Downey DG, Bell SC, Elborn JS: Neutrophils in cystic fibrosis. Thorax 2009;64:81-88.

-7 Russell KJ, McRedmond J, Mukherji N, Costello C, Keatings V, Linnane S, Henry M, Fitzgerald MX, O'Connor CM: Neutrophil adhesion molecule surface expression and responsiveness in cystic fibrosis. Am J Respir Crit Care Med 1998;157:756-761.

$>_{8}$ Witko-Sarsat V, Halbwachs-Mecarelli L, Sermet-Gaudelus I, Bessou G, Lenoir G, Allen RC, Descamps-Latscha B: Priming of blood neutrophils in children with cystic fibrosis: correlation between functional and phenotypic expression of opsonin receptors before and after platelet-activating factor priming. J Infect Dis 1999;179:151-162.

$\checkmark 9$ Adib-Conquy M, Pedron T, Petit-Bertron AF, Tabary O, Corvol H, Jacquot J, Clement A, Cavaillon JM: Neutrophils in cystic fibrosis display a distinct gene expression pattern. Mol Med 2008; 14:36-44.

10 Witko-Sarsat V, Allen RC, Paulais M, Nguyen AT, Bessou G, Lenoir G, Descamps-Latscha B: Disturbed myeloperoxidase-dependent activity of neutrophils in cystic fibrosis homozygotes and heterozygotes, and its correction by amiloride. J Immunol 1996;157: 2728-2735.

-11 Sermet-Gaudelus I, Dechaux M, Vallee B, Fajac A, Girodon E, Nguyen-Khoa T, Marianovski R, Hurbain I, Bresson JL, Lenoir G, Edelman A: Chloride transport in nasal ciliated cells of cystic fibrosis heterozygotes. Am J Respir Crit Care Med 2005;171:1026-1031.

$\checkmark 12$ Rossi AG, Hallett JM, Sawatzky DA, Teixeira MM, Haslett C: Modulation of granulocyte apoptosis can influence the resolution of inflammation. Biochem Soc Trans 2007;35: 288-291.

$\checkmark 13$ Hallett JM, Leitch AE, Riley NA, Duffin R, Haslett C, Rossi AG: Novel pharmacological strategies for driving inflammatory cell apoptosis and enhancing the resolution of inflammation. Trends Pharmacol Sci 2008; 29:250-257.

- 14 Moriceau S, Kantari C, Mocek J, Davezac N, Gabillet J, Guerrera IC, Brouillard F, Tondelier D, Sermet-Gaudelus I, Danel C, Lenoir G, Daniel S, Edelman A, Witko-Sarsat V: Coronin-1 is associated with neutrophil survival and is cleaved during apoptosis: potential implication in neutrophils from cystic fibrosis patients. J Immunol 2009; 182:72547263.
15 McKeon DJ, Condliffe AM, Cowburn AS, Cadwallader KC, Farahi N, Bilton D, Chilvers ER: Prolonged survival of neutrophils from patients with delta F508 CFTR mutations. Thorax 2008;63:660-661.

16 Dibbert B, Weber M, Nikolaizik WH, Vogt $\mathrm{P}$, Schoni MH, Blaser K, Simon HU: Cytokinemediated Bax deficiency and consequent delayed neutrophil apoptosis: a general mechanism to accumulate effector cells in inflammation. Proc Natl Acad Sci USA 1999; 96:13330-13335.

17 Kantari C, Pederzoli-Ribeil M, Amir-Moazami O, Gausson-Dorey V, Moura IC, Lecomte MC, Benhamou M, Witko-Sarsat V: Proteinase 3, the Wegener autoantigen, is externalized during neutrophil apoptosis: evidence for a functional association with phospholipid scramblase 1 and interference with macrophage phagocytosis. Blood 2007;110: 4086-4095.

18 Pardo J, Urban C, Galvez EM, Ekert PG, Muller U, Kwon-Chung J, Lobigs M, Mullbacher A, Wallich R, Borner C, Simon MM: The mitochondrial protein Bak is pivotal for gliotoxin-induced apoptosis and a critical host factor of Aspergillus fumigatus virulence in mice. J Cell Biol 2006;174:509-519.

19 Ward C, Chilvers ER, Lawson MF, Pryde JG, Fujihara S, Farrow SN, Haslett C, Rossi AG: NF- $\kappa \mathrm{B}$ activation is a critical regulator of human granulocyte apoptosis in vitro. J Biol Chem 1999;274:4309-4318.

20 Scheel-Toellner D, Wang K, Craddock R, Webb PR, McGettrick HM, Assi LK, Parkes N, Clough LE, Gulbins E, Salmon M, Lord JM: Reactive oxygen species limit neutrophil life span by activating death receptor signaling. Blood 2004;104:2557-2564.

21 O’Neill AJ, O'Neill S, Hegarty NJ, Coffey RN, Gibbons N, Brady H, Fitzpatrick JM, Watson RW: Glutathione depletion-induced neutrophil apoptosis is caspase 3 dependent. Shock 2000;14:605-609.

22 Rossi AG, Sawatzky DA, Walker A, Ward C, Sheldrake TA, Riley NA, Caldicott A, Martinez-Losa M, Walker TR, Duffin R, Gray M, Crescenzi E, Martin MC, Brady HJ, Savill JS, Dransfield I, Haslett C: Cyclin-dependent kinase inhibitors enhance the resolution of inflammation by promoting inflammatory cell apoptosis. Nat Med 2006;12:1056-1064.

23 Koedel U, Frankenberg T, Kirschnek S, Obermaier B, Häcker H, Paul R, Häcker G: Apoptosis is essential for neutrophil functional shutdown and determines tissue damage in experimental pneumococcal meningitis. PLoS Pathog 2009;5:e1000461.
24 Painter RG, Valentine VG, Lanson NA Jr, Leidal K, Zhang Q, Lombard G, Thompson C, Viswanathan A, Nauseef WM, Wang G, Wang G: CFTR expression in human neutrophils and the phagolysosomal chlorination defect in cystic fibrosis. Biochemistry 2006; 45:10260-10269.

25 Ma T, Thiagarajah JR, Yang H, Sonawane ND, Folli C, Galietta LJ, Verkman AS: Thiazolidinone CFTR inhibitor identified by high-throughput screening blocks cholera toxin-induced intestinal fluid secretion. J Clin Invest 2002;110:1651-1658.

-26 Gottlieb RA, Dosanjh A: Mutant cystic fibrosis transmembrane conductance regulator inhibits acidification and apoptosis in C127 cells: possible relevance to cystic fibrosis. Proc Natl Acad Sci USA 1996;93:35873591.

-27 Jungas T, Motta I, Duffieux F, Fanen P, Stoven V, Ojcius DM: Glutathione levels and Bax activation during apoptosis due to oxidative stress in cells expressing wild-type and mutant cystic fibrosis transmembrane conductance regulator. J Biol Chem 2002; 277:27912-27918.

-28 Hudson VM: Rethinking cystic fibrosis pathology: the critical role of abnormal reduced glutathione (GSH) transport caused by CFTR mutation. Free Radic Biol Med 2001;30:1440-1461.

29 Linsdell P, Hanrahan JW: Glutathione permeability of CFTR. Am J Physiol 1998; 275:C323-C326.

30 Watson RW: Redox regulation of neutrophil apoptosis. Antioxid Redox Signal 2002;4:97104.

- 31 Fadeel B, Ahlin A, Henter JI, Orrenius S, Hampton MB: Involvement of caspases in neutrophil apoptosis: regulation by reactive oxygen species. Blood 1998;92:4808-4818.

32 Konstan MW, Byard PJ, Hoppel CL, Davis PB: Effect of high-dose ibuprofen in patients with cystic fibrosis. N Engl J Med 1995;332: 848-854.

33 Balfour-Lynn IM, Lees B, Hall P, Phillips G, Khan M, Flather M, Elborn JS: Multicenter randomized controlled trial of withdrawal of inhaled corticosteroids in cystic fibrosis. Am J Respir Crit Care Med 2006;173:1356-1362.

34 Gu Y, Harley IT, Henderson LB, Aronow BJ, Vietor I, Huber LA, Harley JB, Kilpatrick JR, Langefeld CD, Williams AH, Jegga AG, Chen J, Wills-Karp M, Arshad SH, Ewart SL, Thio CL, Flick LM, Filippi MD, Grimes HL, Drumm ML, Cutting GR, Knowles MR, Karp CL: Identification of IFRD1 as a modifier gene for cystic fibrosis lung disease. $\mathrm{Na}$ ture 2009;458:1039-1042. 